\title{
Naringenin attenuates fibroblast activation and inflammatory response in a mechanical stretch-induced hypertrophic scar mouse model
}

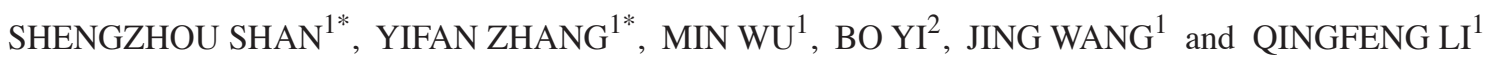 \\ ${ }^{1}$ Department of Plastic and Reconstructive Surgery, Shanghai Ninth People's Hospital, \\ Shanghai Jiao Tong University School of Medicine, Shanghai 200011; ${ }^{2}$ Clinical College of General Hospital of \\ Beijing Military Region, Anhui Medical University, Hefei, Anhui 230000, P.R. China
}

Received April 29, 2016; Accepted March 23, 2017

DOI: $10.3892 / \mathrm{mmr} .2017 .7209$

\begin{abstract}
The pathogenesis and therapy of hypertrophic scars (HS) have not yet been established. The aim of the present study was to investigate the potential effect of naringenin on HS and its underlying mechanisms. The mouse model of HS was prepared by a mechanical stretch device and then treated with naringenin at various concentrations. Histological studies were performed to evaluate scar hypertrophy by hematoxylin and eosin, as well as Masson's trichrome staining. The activation of HS fibroblasts was determined based on reverse transcription-quantitative polymerase chain reaction (RT-qPCR), western blotting and immunohistochemical staining. Following observing the retention of inflammation cells by immunohistochemistry, the cytokines, including tumor necrosis factor (TNF)- $\alpha$, interleukin (IL)- $1 \beta$, IL-6 and transforming growth factor (TGF)- $\beta 1$, mRNA and protein levels were quantitated by RT-qPCR, ELISA and western blotting methods. As a result, naringenin significantly inhibited the formation of HS in a concentration-dependent manner. In addition, naringenin inhibited fibroblast activation and inflammatory cell recruitment. In addition, mRNA and protein expression levels of TNF- $\alpha$, IL- $1 \beta$, IL- 6 and TGF- $\beta 1$ were downregulated following naringenin treatment. The current study highlighted a new pharmacological activity of naringenin on HS. The mechanism of action of naringenin was associated with the inhibition of fibroblast activation and local
\end{abstract}

Correspondence to: Dr Qingfeng Li or Dr Jing Wang, Department of Plastic and Reconstructive Surgery, Shanghai Ninth People's Hospital, Shanghai Jiao Tong University School of Medicine, 639 Zhizaoju Road, Shanghai 200011, P.R. China

E-mail: drliqingfeng@yahoo.com

E-mail: 0127239252@sjtu.edu.cn

*Contributed equally

Key words: naringenin, hypertrophic scar, fibroblast, inflammatory cell, inflammatory cytokine inflammation. These results suggested that naringenin may serve as a novel agent for treatment of HS.

\section{Introduction}

Cutaneous injury triggers an immediate and cascade of repair events that can be summarized as a normal healing response which culminates in scar formation (1). However, aberrant wound healing process results in abnormal scar formation such as hypertrophic scar (HS), which is red, raised and frequently itchy, resulting in functional and cosmetic defects and mental pressure $(2,3)$. Currently, there is no effective treatment for HS, and $\sim 35 \%$ of surgical skin wounds give rise to HS following 1 year (4). Therefore, a valid approach for HS treatment is urgently required for development.

The exact mechanism of HS formation remains unclear. However, potential factors thought to serve a dominant role in human HS formation include mechanical force (5), local inflammation (6,7) and fibroblast activation (8). The exaggerated inflammatory phase is closely associated with HS development, in which inflammatory cells such as neutrophils, macrophages and T-lymphocytes migrate to the wound site with high secretion of various cytokines, including tumor necrosis factor (TNF)- $\alpha$, interleukin (IL)-1 $\beta$, IL-6 and transforming growth factor (TGF)- $\beta 1(7,9,10)$. Specific cytokines and inflammatory factors, such as TGF- $\beta 1$, contribute to fibroblast activation and the modulation of the fibroblast phenotype (11). Many studies have reported that overactivation of dermal fibroblasts is involved in the process of HS formation $(7,12)$. Activated fibroblasts transdifferentiate into myofibroblasts, which contribute to the accumulation of collagens that are responsible for fibrosis (13). Simultaneously, in addition to their profibrotic properties, activated fibrocytes are reported to produce proinflammatory mediators such as IL-6 and IL-8, which participate in the early events mediating inflammation (14). Therefore, inflammation events and fibroblast activation are potential therapeutic targets for the treatment of HS. Since the mid-1960s, intralesional corticosteroid injection has been crucial to treating HS (2) through decreasing the severe inflammatory state in the wound (15). Nonetheless, side effects, for example telangiectasia, rebound 
effects, skin atrophy, hypopigmentation, injection pain and ineffectiveness occur at high frequency (16). Furthermore, there are currently no valid treatment methods available for HS that prevent fibroblast activation. Thus, it is crucial to develop a more effective strategy to regulate this inflammatory process, as well as fibroblast activation to prevent hypertrophic scarring.

As a natural predominant flavanone, naringenin (4',5,7-trihydroxyflavanone) has an extensive scope of pharmacological characteristics, such as anti-tumor (17), antimutagenic (18) and anti-atherogenic (19) activity. Furthermore, naringenin has been proved to possess anti-inflammatory effects in a number of illnesses. Naringenin has greatly reduced the symptom of colitis, down regulating the pro-inflammatory mediators, for instance, IL-6 and TNF- $\alpha$ in colon mucosa (20). It has been suggested that naringenin demonstrates anti-inflammatory effect on chronic bronchitis by reducing the concentrations of IL-8, leukotriene B4 and TNF- $\alpha$ in bronchoalveolar lavage fluid (21). In addition, naringenin downregulates the release of proinflammatory cytokines such as TNF- $\alpha$ and IL- $1 \beta$, which abrogated the ischemic brain injury through the suppression of nuclear factor- $\kappa \mathrm{B}$-mediated neuroinflammation (22). Conversely, it has been proven to attenuate interstitial fibrosis in pressure induced-cardiac hypertrophy via PI3K/Akt, ERK and JNK signaling pathways (23) and to inhibit renal fibrosis by blocking Smad3 phosphorylation and transcription (24).

Given these evidences, the authors hypothesize about what effect naringenin has on dermal fibroblasts, and whether naringenin could inhibit dermal inflammation and HS formation in vivo. Consequently, the present study investigated its function in the HS model induced by mechanical load. Furthermore, its effects on distinct aspects of local inflammation are investigated, such as inflammatory cell infiltration and inflammatory cytokine release, and tested its effects on fibroblasts activation in vivo.

\section{Materials and methods}

Animals and HS model. Shanghai SLAC Laboratory Animal Co., Ltd. (Shanghai, China) provided 24 female KM mice, 8-weeks-old and 30-40 g for the experiment. The School of Medicine, Shanghai Jiao Tong University Animal Care and Use Committee (Shanghai, China) approved the entire process and all the animals were kept in standardized conditions under guidelines approved by the institution. They had a $14 \mathrm{~h}$ light/10 h dark light cycle and had free access to food and water. Based on a published approach, the model of HS was built. Briefly, the mice were anaesthetized by $4 \%$ chloral hydrate intraperitoneal injection $(0.1 \mathrm{ml} / 10 \mathrm{~g})$ and a $2 \mathrm{~cm}$ incision was made and sewed into the dorsal midline. At day 4 , the sutures were removed. Mechanical stretch devices were sewed, covering the incisions, and, following the incision, stretch was conducted from day 4 to 14 . The animals were grouped into three categories at random with eight mice in every group. Group A was the control group, being administered with 10\% dimethylsulfoxide (DMSO; Sigma-Aldrich; Merck KGaA, Darmstadt, Germany) in sterile saline every day. Following incision, Groups $\mathrm{B}$ and $\mathrm{C}$ were the model of HS, being administered with naringenin (dissolved in DMSO) at a concentration of $25 \mu \mathrm{M}$ and a concentration of $50 \mu \mathrm{M}$ in group $\mathrm{B}$ and $\mathrm{C}$ respectively for exterior usage of once daily from day 4 to 14 . On day 14 (24 h following the last usage of drug), all the mice were sacrificed by cervical dislocation and the specimens were collected for future study.

Histology and immunohistochemistry. The $5 \mu \mathrm{m}$ paraffinembedded and paraformaldehyde-fixed (4\% paraformaldehyde overnight) tissue sections were stained with hematoxylin and eosin (H\&E) and Masson's trichrome (Trichrome stain LG solution, HT10316; Sigma-Aldrich; Merck KGaA). Regarding immunohistochemical staining, the sections were found with primary antibodies against $\alpha$-smooth muscle actin (SMA; ab5694; 1:200; Abcam, Cambridge, MA, USA), CD4 (ab183685; 1:200; Abcam), CD68 (ab125212; 1:200; Abcam) at $4^{\circ} \mathrm{C}$ overnight. Following incubation with the peroxidase-conjugated secondary antibody (KIT-5004; original concentration; Fuzhou Maixin Biotech Co., Ltd., Fuzhou, China) for $15 \mathrm{~min}$ at room temperature, the sections were counterstained with hematoxylin and developed with diaminobenzidine. The images were processed for publication purpose using Adobe Photoshop CS4 (Adobe Systems, Inc., San Jose, CA, USA).

Reverse transcription-quantitative polymerase chain reaction (RT-qPCR). The scar tissue of the mice (50-100 mg) was thoroughly ground in liquid nitrogen with a pestle and mortar, and then evenly divided with a syringe and needle. According to the manufacturer's instructions, TRIzol reagent (Invitrogen; Thermo Fisher Scientific, Inc., Waltham, MA, USA) was used to isolate total RNA which was subjected to reverse transcription with M-MLV Reverse Transcriptase (Thermo Fisher Scientific, Inc.) and oligodeoxynucleotide (Sangon Biotech Co., Ltd., Shanghai, China). RNA concentration and purity was measured according to the $260 \mathrm{~nm}$ absorbance and the rate of absorbance at 260/280 nm on NanoDrop2000 spectrophotometer (Thermo Fisher Scientific, Inc.). Synthesized cDNA was analyzed with RT-qPCR using SYBR ${ }^{\circledR}$ Premix (Takara Biotechnology Co., Ltd., Dalian, China) and the 7900 HT Fast real-time q-PCR system (Thermo Fisher Scientific, Inc.). RT-qPCR cycling conditions were as follows: 40 cycles each involving $5 \mathrm{sec}$ of denaturation at $95^{\circ} \mathrm{C}$ and $30 \mathrm{sec}$ of annealing/extension at $60^{\circ} \mathrm{C}$. GAPDH was utilized as a reference gene. The gene expression data were analyzed using the $2^{-\Delta \Delta \mathrm{Cq}}$ method (25). Primers sequences used are as below: $\alpha$-SMA forward, 5-GTCCCAGACATCAGGGAGTAA-3 and reverse, 5-TCGGATACTTCAGCGTCAGGA-3; IL-1 $\beta$ forward 5-GAAATGCCACCTTTTGACAGTG-3, and reverse, 5-TGG ATGCTCTCATCAGGACAG-3; IL-6 forward, 5-TCTATA CCACTTCACAAGTCGGA-3 and reverse, 5-GAATTGCCA TTGCACAACTCTTT-3; TGF- $\beta 1$ forward, 5-CCACCTGCA AGACCATCGAC-3 and reverse, 5-CTGGCGAGCCTTAGT TTGGAC-3; TNF- $\alpha$ forward, 5-CAGGCGGTGCCTATG TCTC-3 and reverse 5-CGATCACCCCGAAGTTCAGTAG-3. Each experiment was replicated three times.

Western blot analysis. Total proteins were extracted from skin scar tissues utilizing radioimmunoprecipitation assay lysis buffer (Shanghai Shenergy Biocolor BioScience \& Technology Company, Shanghai, China). The bicinchoninic 
A
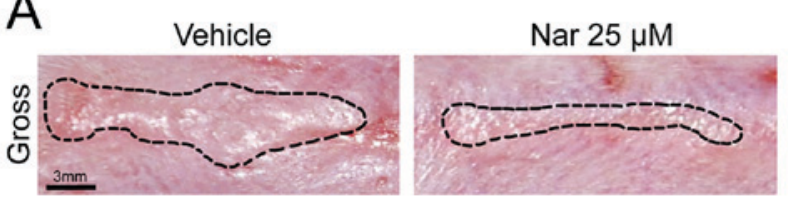

B

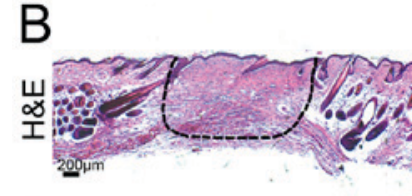

C
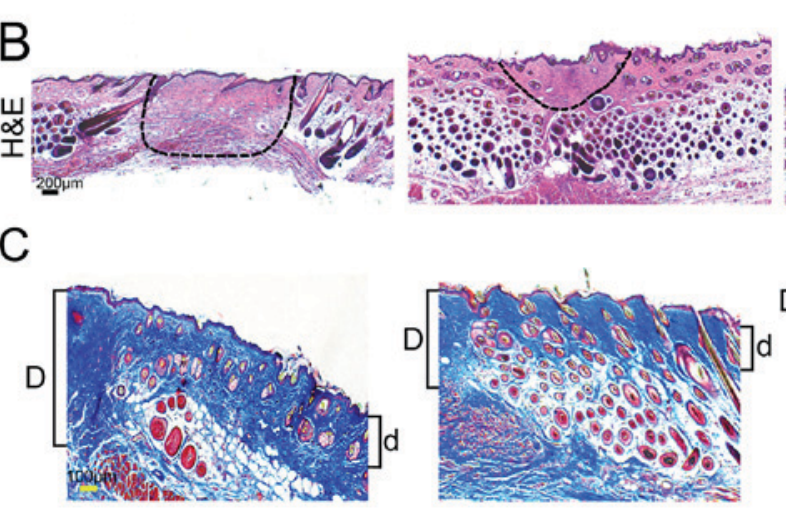
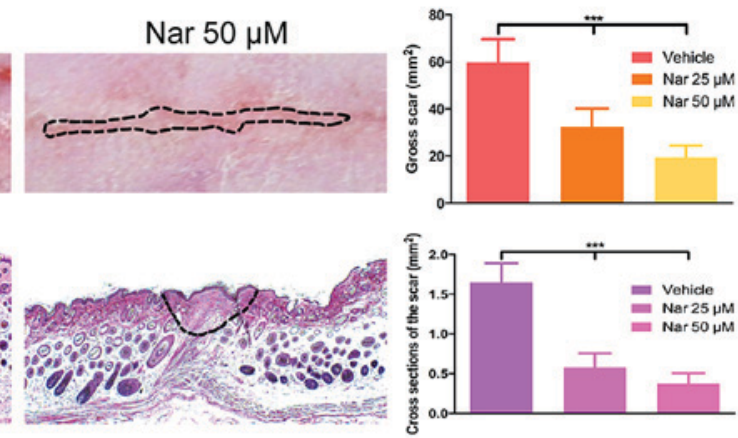

sos
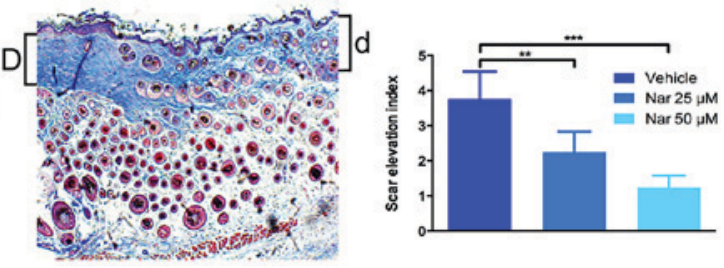

Figure 1. Decreased scar formation in Nar-treated mouse. (A) Inhibition effects of Nar on scar hypertrophy by images of gross scars sections. (B) H\&E stained sections and (C) Masson's trichrome stained sections. SEI was measured. 'D' and 'd' serve as the thickness of the scar and bordering normal skin, respectively. SEI is defined as the D/d ratio. The dotted lines draft the scar. Data are expressed as mean \pm standard deviation of eight mice per group. Vehicle, dimethylsulfoxide control. ${ }^{* *} \mathrm{P}<0.01,{ }^{* * *} \mathrm{P}<0.001$. Nar, naringenin; SEI, scar elevation index; H\&E, hematoxylin and eosin.

acid assay (Thermo Fisher Scientific, Inc.) was used to determine the concentration of protein. The proteins at the dose of $20 \mu \mathrm{g}$ were isolated by $10 \%$ SDS-PAGE and electroblotted in the membranes of polyvinylidene difluoride (EMD Millipore, Bedford, MA, USA). The aspecific protein binding sites on the membranes were blocked with 5\% BSA (Biosharp, Hefei, China). Then the membranes were gently agitated and incubated with antibodies against IL-1 $\beta$ (ab9722; 1:500; Abcam), IL-6 (ab208113; 1:500; Abcam), TGF- $\beta 1$ (ab92486; 1:500; Abcam), TNF- $\alpha$ (ab6671; 1:500; Abcam), GAPDH (AP0063; 1:10,000; Bioworld Technology, Inc., St. Louis Park, MN, USA;), and $\alpha$-SMA (ab5694; 1:500; Abcam) at $4^{\circ} \mathrm{C}$ overnight. The following day, the membranes were incubated with peroxidase-conjugated anti-rabbit IgG secondary antibody (7074S; 1:1,000; Cell Signaling Technology, Inc., Danvers, MA, USA). The bands were visualized using an enhanced chemiluminescence detection system (EMD Millipore, Billerica, MA, USA) and quantitative analysis was conducted using Image $\mathbf{J}$ software version 1.4.3.67 (National Institutes of Health, Bethesda, MD, USA).

ELISA. Based on the instructions of the manufacturer, the expression degrees of IL- $1 \beta$, IL- 6 , TGF- $\beta 1$ and TNF- $\alpha$ in the extract of mouse skin scar tissue ( $n=8$, each group) were identified by ELISA kits (IL-1 $\beta$, MLB00C; IL-6, M6000B; TNF- $\alpha$, MTA00B; TGF- $\beta 1$, MB100B all from the R\&D Systems, Inc., Minneapolis, MN, USA). According to the color reaction of antibodies and cytoplasm extract, the values of absorbance can be identified at $450 \mathrm{~nm}$ on a microplate reader (Tecan Group Ltd., Männedorf, Switzerland).

Statistical analysis. All values were expressed as means \pm standard deviation. Analysis of data was conducted using post-hoc least significant difference test and Friedman's analysis of variance. $\mathrm{P}<0.05$ was considered to indicate a statistically significant difference.

\section{Results}

Naringenin attenuated scar formation. Initially, the present study investigated whether topical application of naringenin have anti-fibrotic effects on the HS mouse model during scar formation, mice were treated with naringenin at the doses of 25 and $50 \mu \mathrm{M}$. Following exterior use of naringenin for 10 days (from day 4 to day 14 following incision), in comparison to the vehicle group of DMSO-treated mice, the groups with the treatment of naringenin presented greatly decreased formation of scar with dose-dependency. As demonstrated in Fig. 1A, the gross scar section area decreased in varying degrees following the treatment of naringenin. Alternatively, H\&E staining and Masson's trichrome staining showed that cross section of the scar and scar elevation index decreased, respectively (Fig. 1B and $\mathrm{C}$ ). These results indicated that topical application of naringenin on the HS mouse model had antifibrotic effects and inhibited scar formation induced by mechanical stretch.

Naringenin inhibited fibroblast activation. The degrees of $\alpha$-SMA expression in the scar section of the HS mouse model were evaluated in order to investigate the effect of naringenin on fibroblast activation. Compared with vehicle group, $\alpha$-SMA expression was considerably decreased in the fibrotic tissues of naringenin-treated mice with dose dependency. According to the study, the mRNA expression and protein production of $\alpha$-SMA in the model of HS mouse were greatly reduced by the treatment of naringenin (Fig. 2A and B). Furthermore, the cells were immunostained for the presence of $\alpha$-SMA. In addition, the rate of $\alpha$-SMA positive cells was much higher in vehicle mouse sections than that in the scar sections of naringenin-treated mice (Fig. 2C). These findings demonstrated that the activation of fibroblasts induced by mechanical stretch and injury on the model of HS mouse could be inhibited by naringenin administration. 

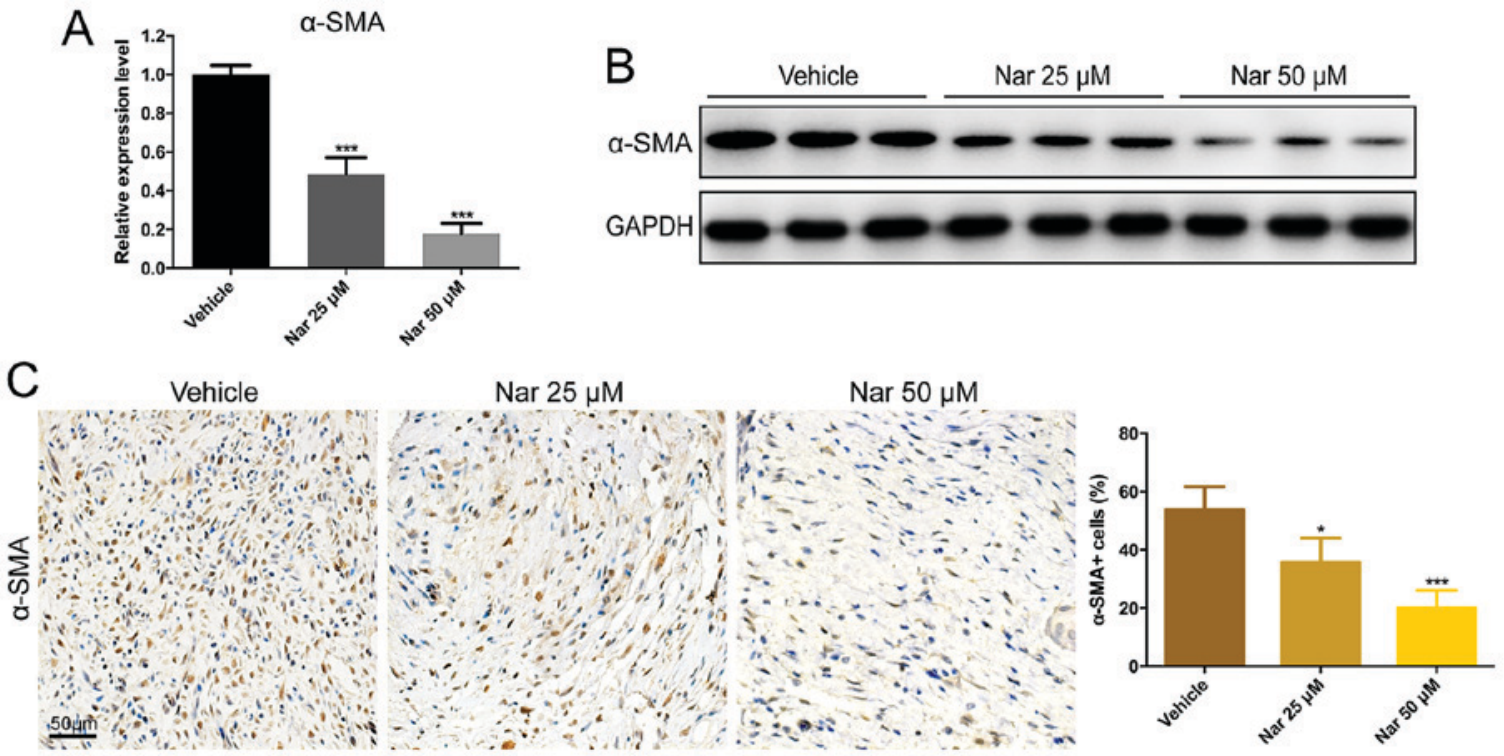

Figure 2. Decreased activation of fibroblast in Nar-treated mouse scar tissues. Inhibition effects of Nar on $\alpha$-SMA by (A) reverse transcription-quantitative polymerase chain reaction, (B) western blotting and (C) immunohistochemical staining. Data are expressed as mean \pm standard deviation of eight mice per group. ${ }^{*} \mathrm{P}<0.05,{ }^{* * *} \mathrm{P}<0.001$ vs. Vehicle. Vehicle, dimethylsulfoxide control. SMA, smooth muscle actin; Nar, naringenin.

Naringenin suppressed inflammatory cell infiltration. To study the effects of naringenin on inflammatory cell, cells were immunostained for the presence of CD68+ cells and CD4+ cells. Immunohistochemistry data indicated that, in comparison to the vehicle group, the CD68+ cells and CD4+ cells in the scar tissues of a naringenin-treated mouse were greatly decreased with dose dependency (Fig. 3). This result indicated that the inflammatory response could be suppressed by naringenin through decreasing the infiltration of inflammatory cells on the model of the HS mouse.

Naringenin reduced the expression of inflammatory cytokine. To investigate the effects of naringenin on inflammatory cytokines, IL-1 $\beta$, IL-6, TGF- $\beta 1$ and TNF- $\alpha$ gene expression was observed by RT-qPCR. As a result, IL-1 $\beta$, IL-6, TGF- $\beta 1$ and TNF- $\alpha$ in naringenin-treated mouse scar tissues were significantly reduced at the concentration dose of 25 and $50 \mu \mathrm{M}$ as compared with vehicle group (Fig. 4A). In addition, the protein expression level detected by ELISA and western blotting indicated that naringenin significantly downregulated IL-1 $\beta$, IL-6, TGF- $\beta 1$ and TNF- $\alpha$ expression in a dose-dependent manner in the HS mouse model, respectively (Fig. 4B and C). Collectively, these data suggested that naringenin treatment on HS mouse model reduced infiltration of inflammatory cell and production of inflammatory cytokines.

\section{Discussion}

HS is a complex fibro-proliferative disorder and its definitive mechanism is still not clearly understood. Various invasive and non-invasive options are currently available for the prevention and treatment of HS. The invasive methods include corticosteroid intralesional injections, cryotherapy, laser treatment, radiation therapy and surgical excision (26). In contrast, the non-invasive methods that are easy and comfortable to use and low in expenses are becoming more and more popular. A growing body of evidence proves that the compression therapy is effective in healing scars $(27,28)$. However, pressure therapy also has some defects, such as the use limitation in bending, anatomic compression, or fast-moving regions. Furthermore, as patients wear clothes all the time, they may feel uncomfortable or even suffer from skin ulcers due to uneven pressure distribution (29). Therefore, a vital problem may lie in patients' noncompliance, which is reported to be $8.5-59 \%$ (30). Silicone gel sheeting has been widely used in clinics for the treatment of HS since early 1980s (3), and it is effective in preventing HS according to several researches $(31,32)$. Nevertheless, according to a systematic review published in 2013, there was no sufficient evidence to prove the efficacy of silicone gel sheeting in preventing high-risk individuals from abnormal scarring (33). In addition, silicone has many unavoidable shortcomings, including excessive sweating, skin maceration and itching $(34,35)$, and its accurate mechanism is not understood. Another product for treating scars is onion extract, which is believed to exhibit anti-inflammatory, bacteriostatic and collagen down-regulatory properties (36) and improve collagen formulation in rabbit ear models (37). However, according to clinical research data, onion extract is not able to improve hypertrophic scarring $(38,39)$. Accordingly, compounds to treat HS with less side effects and optimized treatment methods should be determined. In the authors' research, it was observed that topical administration of naringenin suppressed HS development without systemic health damage, and the drug-delivery greatly alleviated the pain caused by traditional intralesional injection (40). Thus, the authors estimate that local application of naringenin is a possible optimum method for treating HS.

The fibroblasts are crucial cells during scarring. However, the fibroblasts that are inactive when the skin is in a good condition will be activated once the skin is hurt (41). Different from scarless healing, the constant activation of fibroblasts is maintained in abnormal scars (42). In the process of HS formation, 


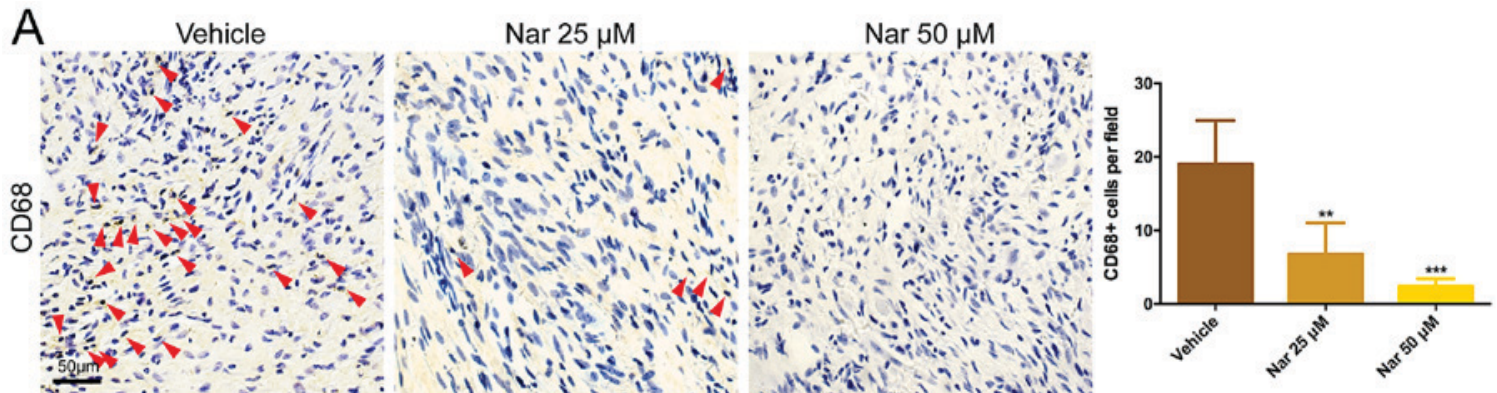

B

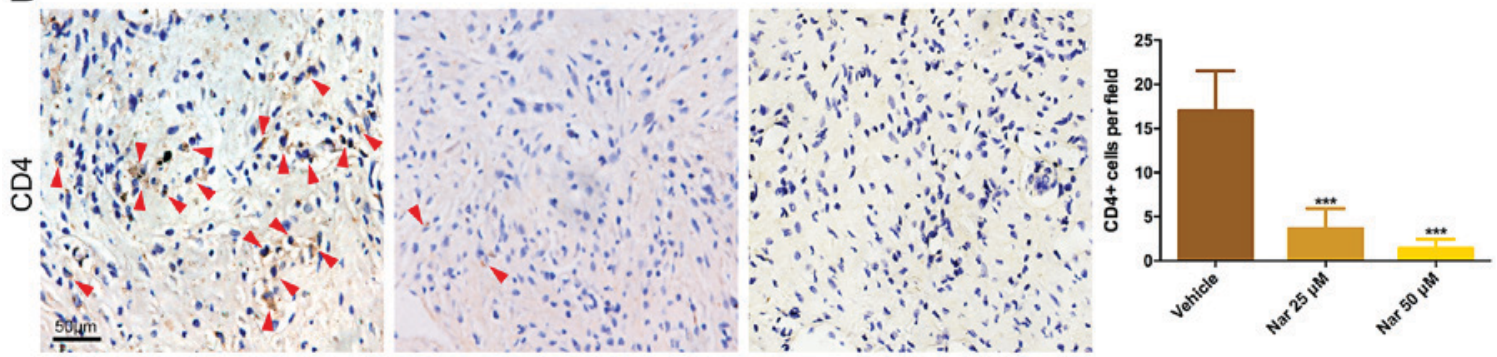

Figure 3. Decreased infiltration of CD68+ and CD4+ cells in Nar-treated mouse scar tissues. Inhibition effects of Nar on (A) CD68+ and (B) CD4+ cells infiltration by immunohistochemical staining. Red arrows indicate positive cells. Data are expressed as mean \pm standard deviation of eight mice per group. ${ }^{* *} \mathrm{P}<0.01 .{ }^{* * *} \mathrm{P}<0.001$ vs. Vehicle. Vehicle, dimethylsulfoxide control. Nar, naringenin.
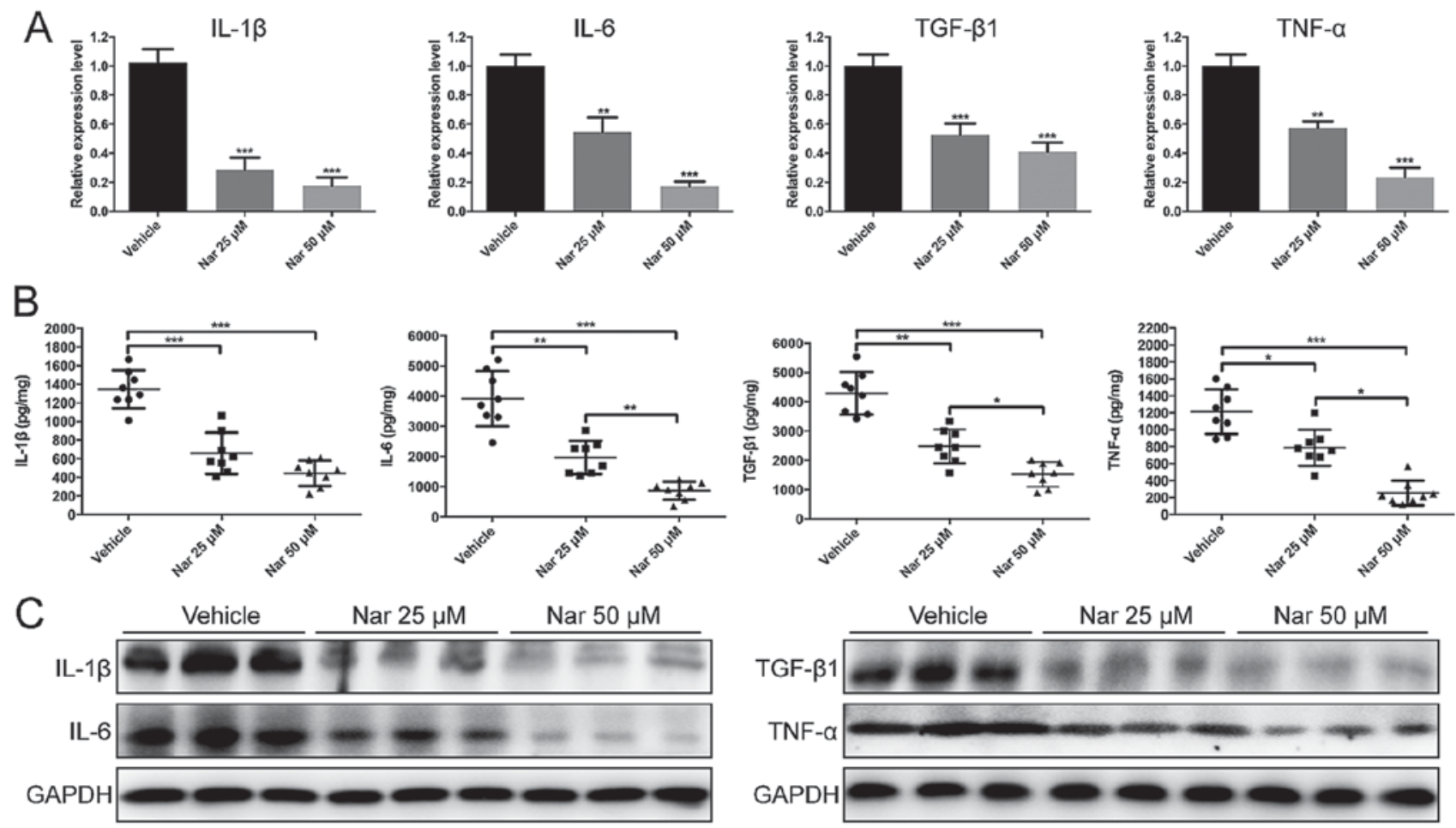

Figure 4. Decreased production of IL-1 $\beta$, IL-6, TGF- $\beta 1$ and TNF- $\alpha$ in Nar-treated mouse scar tissues. Effects of Nar on the mRNA and protein expressions of IL-1 $\beta$, IL-6, TGF- $\beta 1$ and TNF- $\alpha$ were determined by (A) reverse transcription-quantitative polymerase chain reaction, (B) ELISA and (C) western blotting. Data are expressed as mean \pm standard deviation of eight mice per group. ${ }^{*} \mathrm{P}<0.05,{ }^{* *} \mathrm{P}<0.01,{ }^{* * *} \mathrm{P}<0.001$. DMSO, dimethylsulfoxide control. IL, interleukin; TGF, transforming growth factor; TNF, tumor necrosis factor; Nar, naringenin.

fibroblasts are unduly stimulated and trans-differentiate into myofibroblasts $(43,44)$. Myofibroblasts express excessive $\alpha$-SMA (45), which is closely related to scar contracture and texture $(41,46)$, and produce extracellular matrix proteins $(47)$. Therefore, fibroblasts have become the possible therapeutic target for antifibrosis treatment. Previously, naringenin has been reported to show antifibrosis outcomes in several fibrotic diseases, such as lung fibrosis (48) and liver fibrosis (49). To the best of authors' knowledge, the present study is the first to investigate effects of naringenin on fibroblasts derived from HS. Results demonstrated that the mRNA and protein levels of $\alpha$-SMA in naringenin-treated scars were lower, thus 
demonstrating that naringenin could decrease scar formation by inhibiting HS-derived fibroblast activation.

Inflammation clearly serves a major role in HS progression. Compared with the normal scarring, HS presents an enhanced and prolonged inflammation phase characterized by a larger number of inflammatory cells recruitment and inflammatory cytokine infiltration $(6,7,10)$. It is reported that macrophages $(50)$ and lymphocytes, especially CD4+ T lymphocytes $(51,52)$, are the dominant inflammatory cells during HS pathology. These inflammatory cells prompt exaggerated scar formation by modulating the fibroblast phenotype and secreting cytokines such as IL-1 $\beta$, IL-6, TGF- $\beta 1$ and TNF- $\alpha$. Elevated levels of proinflammatory cytokines (IL-1 $\beta$, IL- 6 and TNF- $\alpha$ ) have been observed in HS $(9,10)$. Herein, the authors demonstrated that treatment with naringenin inhibited the CD4+ T lymphocyte and CD68+ cells (monocyte/macrophage) retention, as well as proinflammatory cytokines levels, such as IL- $1 \beta$, IL- 6 and TNF- $\alpha$. Moreover, naringenin inhibited TGF- $\beta 1$ production in HS. In the cases of acute inflammation, TGF- $\beta 1$ belongs to anti-inflammatory cytokines, which are generated together with the pro-inflammatory cytokines and used to inhibit inflammatory reactions (53). In this context, the suppression of IL-1 $\beta$, IL- 6 and TNF- $\alpha$ production may result in the reduction of TGF- $\beta 1$ levels.

In conclusion, the current research indicated that topical application of naringenin to hypertrophic scar mouse model can suppress HS formation. Further analyses suggests that naringenin has effectively inhibits trans-differentiation of fibroblasts into myofibroblasts, suppresses inflammatory cell infiltration, as well as downregulates the expression of inflammatory cytokines. Therefore, naringenin, with its inhibitory effects on fibroblast activation and local inflammation and relatively low cost and toxicity of natural agents, could be used as a safe, alternative therapeutic agent for treating HS. However, the precise underlying mechanism needs further elucidation.

\section{Acknowledgements}

The present study was supported by grants from the key project of the National Natural Science Foundation (grant no. 81230042) and the National Key Project of Scientific and Technical Supporting Programs Funded by Ministry of Science \& Technology of China (grant no. 2012BAI11B03).

\section{References}

1. Bran GM, Goessler UR, Hormann K, Riedel F and Sadick H: Keloids: Current concepts of pathogenesis (Review). Int J Mol Med 24: 283-293, 2009.

2. Chen MA and Davidson TM: Scar management: Prevention and treatment strategies. Curr Opin Otolaryngol Head Neck Surg 13: 242-247, 2005

3. Mustoe TA, Cooter RD, Gold MH, Hobbs FD, Ramelet AA, Shakespeare PG, Stella M, Téot L, Wood FM and Ziegler UE; International Advisory Panel on Scar Management: International clinical recommendations on scar management. Plast Reconstr Surg 110: 560-571, 2002.

4. Butzelaar L, Ulrich MM, Mink van der Molen AB, Niessen FB and Beelen RH: Currently known risk factors for hypertrophic skin scarring: A review. J Plast Reconstr Aesthet Surg 69: 163-169, 2015.

5. Aarabi S, Bhatt KA, Shi Y, Paterno J, Chang EI, Loh SA, Holmes JW, Longaker MT, Yee H and Gurtner GC: Mechanical load initiates hypertrophic scar formation through decreased cellular apoptosis. FASEB J 21: 3250-3261, 2007.
6. Gurtner GC, Werner S, Barrandon Y and Longaker MT: Wound repair and regeneration. Nature 453: 314-321, 2008.

7. van der Veer WM, Bloemen MC, Ulrich MM, Molema G, van Zuijlen PP, Middelkoop E and Niessen FB: Potential cellular and molecular causes of hypertrophic scar formation. Burns 35 : 15-29, 2009.

8. Sarrazy V, Billet F, Micallef L, Coulomb B and Desmoulière A: Mechanisms of pathological scarring: Role of myofibroblasts and current developments. Wound Repair Regen 19 ( Suppl 1): S10-S15, 2011.

9. Ray S, Ju X, Sun H, Finnerty CC, Herndon DN and Brasier AR: The IL-6 trans-signaling-STAT3 pathway mediates ECM and cellular proliferation in fibroblasts from hypertrophic scar. J Invest Dermatol 133: 1212-1220, 2013.

10. Salgado RM, Alcantara L, Mendoza-Rodriguez CA, Cerbón M, Hidalgo-González C, Mercadillo P, Moreno LM, Alvarez-Jiménez R and Krötzsch E: Post-burn hypertrophic scars are characterized by high levels of IL-1 $\beta$ mRNA and protein and TNF- $\alpha$ type I receptors. Burns 38: 668-676, 2012.

11. Ren Y, Deng CL, Wan WD, Zheng JH, Mao GY and Yang SL: Suppressive effects of induced pluripotent stem cell-conditioned medium on in vitro hypertrophic scarring fibroblast activation. Mol Med Rep 11: 2471-2476, 2015.

12. Zhang YF, Zhou SZ, Cheng XY, Yi B, Shan SZ, Wang J and Li QF: Baicalein attenuates hypertrophic scar formation via inhibition of the transforming growth factor- $\beta / \operatorname{Smad} 2 / 3$ signalling pathway. Br J Dermatol 174: 120-130, 2016.

13. Gauglitz GG, Korting HC, Pavicic T, Ruzicka T and Jeschke MG: Hypertrophic scarring and keloids: Pathomechanisms and current and emerging treatment strategies. Mol Med 17: 113-125, 2011.

14. Peng $\mathrm{H}$ and Herzog EL: Fibrocytes: Emerging effector cells in chronic inflammation. Curr Opin Pharmacol 12: 491-496, 2012.

15. Niessen FB, Spauwen PH, Schalkwijk J and Kon M: On the nature of hypertrophic scars and keloids: A review. Plast Reconstr Surg 104: 1435-1458, 1999.

16. Arno AI, Gauglitz GG, Barret JP and Jeschke MG: Up-to-date approach to manage keloids and hypertrophic scars: A useful guide. Burns 40: 1255-1266, 2014.

17. So FV, Guthrie N, Chambers AF and Carroll KK: Inhibition of proliferation of estrogen receptor-positive MCF-7 human breast cancer cells by flavonoids in the presence and absence of excess estrogen. Cancer lett 112: 127-133, 1997.

18. Francis AR, Shetty TK and Bhattacharya RK: Modulating effect of plant flavonoids on the mutagenicity of N-methyl-N'-nitro-N-nitrosoguanidine. Carcinogenesis 10: 1953-1955, 1989.

19. Lee CH, Jeong TS, Choi YK, Hyun BH, Oh GT, Kim EH, Kim JR, Han JI and Bok SH: Anti-atherogenic effect of citrus flavonoids, naringin and naringenin, associated with hepatic ACAT and aortic VCAM-1 and MCP-1 in high cholesterol-fed rabbits. Biochem Biophys Res Commun 284: 681-688, 2001.

20. Dou W, Zhang J, Sun A, Zhang E, Ding L, Mukherjee S, Wei X, Chou G, Wang ZT and Mani S: Protective effect of naringenin against experimental colitis via suppression of Toll-like receptor 4/NF-kB signalling. Br J Nutr 110: 599-608, 2013.

21. Luo YL, Zhang CC, Li PB, Nie YC, Wu H, Shen JG and Su WW: Naringin attenuates enhanced cough, airway hyperresponsiveness and airway inflammation in a guinea pig model of chronic bronchitis induced by cigarette smoke. Int Immunopharmacol 13: 301-307, 2012.

22. Raza SS, Khan MM, Ahmad A, Ashafaq M, Islam F, Wagner AP, Safhi MM and Islam F: Neuroprotective effect of naringenin is mediated through suppression of NF- $\mathrm{kB}$ signaling pathway in experimental stroke. Neuroscience 230: 157-171, 2013.

23. Zhang N, Yang Z, Yuan Y, Li F, Liu Y, Ma Z, Liao H, Bian Z, Zhang Y, Zhou H, et al: Naringenin attenuates pressure overload-induced cardiac hypertrophy. Exp Ther Med 10: 2206-2212, 2015.

24. Meng XM, Zhang Y, Huang XR, Ren GL, Li J and Lan HY: Treatment of renal fibrosis by rebalancing TGF- $\beta / \mathrm{Smad}$ signaling with the combination of asiatic acid and naringenin. Oncotarget 6: 36984-36997, 2015.

25. Livak KJ and Schmittgen TD: Analysis of relative gene expression data using real-time quantitative PCR and the 2 (-Delta Delta C(T)) method. Methods 25: 402-408, 2001.

26. Meaume S, Le Pillouer-Prost A, Richert B, Roseeuw D and Vadoud J: Management of scars: Updated practical guidelines and use of silicones. Eur J Dermatol 24: 435-443, 2014. 
27. Puzey G: The use of pressure garments on hypertrophic scars J Tissue Viability 12: 11-15, 2002.

28. Yagmur C, Akaishi S, Ogawa R and Guneren E: Mechanical receptor-related mechanisms in scar management: A review and hypothesis. Plast Reconstr Surg 126: 426-434, 2010

29. Zurada JM, Kriegel D and Davis IC: Topical treatments for hypertrophic scars. J Am Acad Dermatol 55: 1024-1031, 2006.

30. Johnson J, Greenspan B, Gorga D, Nagler W and Goodwin C: Compliance with pressure garment use in burn rehabilitation. J Burn Care Rehabil 15: 180-188, 1994.

31. Gold MH: Topical silicone gel sheeting in the treatment of hypertrophic scars and keloids. A dermatologic experience. J Dermatol Surg Oncol 19: 912-916, 1993.

32. Chernoff WG, Cramer H and Su-Huang S: The efficacy of topical silicone gel elastomers in the treatment of hypertrophic scars, keloid scars and post-laser exfoliation erythema. Aesthetic Plast Surg 31: 495-500, 2007.

33. O'Brien L and Jones DJ: Silicone gel sheeting for preventing and treating hypertrophic and keloid scars. Cochrane Database Syst Rev: CD003826, 2013.

34. Fette A: Influence of silicone on abnormal scarring. Plast Surg Nurs 26: 87-92, 2006.

35. Nikkonen MM, Pitkanen JM and Al-Qattan MM: Problems associated with the use of silicone gel sheeting for hypertrophic scars in the hot climate of Saudi Arabia. Burns 27: 498-501, 2001

36. Augusti KT: Therapeutic values of onion (Allium cepa L.) and garlic (Allium sativum L.). Indian J Exp Biol 34: 634-640, 1996

37. Saulis AS, Mogford JH and Mustoe TA: Effect of Mederma on hypertrophic scarring in the rabbit ear model. Plast Reconstr Surg 110: 177-183; 184-176, 2002.

38. Jackson BA and Shelton AJ: Pilot study evaluating topical onion extract as treatment for postsurgical scars. Dermatol Surg 25 267-269, 1999.

39. Chung VQ, Kelley L, Marra D and Jiang SB: Onion extract gel versus petrolatum emollient on new surgical scars: prospective double-blinded study. Dermatol Surg 32: 193-197, 2006.

40. Ledon JA, Savas J, Franca K, Chacon A and Nouri K: Intralesional treatment for keloids and hypertrophic scars: A review. Dermatol Surg 39: 1745-1757, 2013.

41. Hinz B, Phan SH, Thannickal VJ, Galli A, Bochaton-Piallat ML and Gabbiani G: The myofibroblast: One function, multiple origins. Am J Pathol 170: 1807-1816, 2007.
42. Honardoust D, Ding J, Varkey M, Shankowsky HA and Tredget EE: Deep dermal fibroblasts refractory to migration and decorin-induced apoptosis contribute to hypertrophic scarring. J Burn Care Res 33: 668-677, 2012.

43. Desmouliere A, Chaponnier C and Gabbiani G: Tissue repair, contraction and the myofibroblast. Wound Repair Regen 13: 7-12, 2005.

44. Hinz B: Formation and function of the myofibroblast during tissue repair. J Invest Dermatol 127: 526-537, 2007.

45. Tomasek JJ, Gabbiani G, Hinz B, Chaponnier C and Brown RA: Myofibroblasts and mechano-regulation of connective tissue remodelling. Nat Rev Mol Cell Biol 3: 349-363, 2002.

46. Wang XQ, Kravchuk O, Winterford C and Kimble RM: The correlation of in vivo burn scar contraction with the level of alpha-smooth muscle actin expression. Burns 37: 1367-1377, 2011.

47. Zhu Z, Ding J, Shankowsky HA and Tredget EE: The molecular mechanism of hypertrophic scar. J Cell Commun Signal 7: 239-252, 2013.

48. Du G, Jin L, Han X, Song Z, Zhang H and Liang W: Naringenin A potential immunomodulator for inhibiting lung fibrosis and metastasis. Cancer Res 69: 3205-3212, 2009.

49. Liu X, Wang W, Hu H, Tang N, Zhang C, Liang W and Wang M: Smad3 specific inhibitor, naringenin, decreases the expression of extracellular matrix induced by TGF-beta1 in cultured rat hepatic stellate cells. Pharm Res 23: 82-89, 2006.

50. Wynn TA and Barron L: Macrophages: Master regulators of inflammation and fibrosis. Semin Liver Dis 30: 245-257, 2010.

51. Wynn TA: Fibrotic disease and the $\mathrm{T}(\mathrm{H}) 1 / \mathrm{T}(\mathrm{H}) 2$ paradigm. Nat Rev Immunol 4: 583-594, 2004.

52. Wong VW,Paterno J, Sorkin M, Glotzbach JP,LeviK, Januszyk M, Rustad KC, Longaker MT and Gurtner GC: Mechanical force prolongs acute inflammation via T-cell-dependent pathways during scar formation. FASEB J 25: 4498-4510, 2011.

53. Verri WA Jr, Cunha TM, Parada CA, Poole S, Cunha FQ and Ferreira SH: Hypernociceptive role of cytokines and chemokines: Targets for analgesic drug development? Pharmacol Ther 112: $116-138,2006$ 